\title{
Investigating stability of Laplacians on signed digraphs via eventual positivity
}

Claudio Altafini

The self-archived postprint version of this journal article is available at Linköping University Institutional Repository (DiVA):

http:/ / urn.kb.se/ resolve?urn=urn:nbn:se:liu:diva-168921

N.B.: When citing this work, cite the original publication.

Altafini, C., (2019), Investigating stability of Laplacians on signed digraphs via eventual positivity, 2019 IEEE 58th Conference on Decision and Control (CDC).

https:// doi.org/ 10.1109/ CDC40024.2019.9030125

Original publication available at:

https:/ / doi.org/ 10.1109/ CDC40024.2019.9030125

Copyright: IEEE

http:// www.ieee.org/

(C) 2019 IEEE. Personal use of this material is permitted. However, permission to reprint/ republish this material for advertising or promotional purposes or for creating new collective works for resale or redistribution to servers or lists, or to reuse any copyrighted component of this work in other works must be obtained from the IEEE. 


\title{
Investigating stability of Laplacians on signed digraphs via eventual positivity* $^{*}$
}

\author{
Claudio Altafini
}

\begin{abstract}
Signed Laplacian matrices generally fail to be diagonally dominant and may fail to be stable. For both undirected and directed graphs, in this paper we present conditions guaranteeing the stability of signed Laplacians based on the property of eventual positivity, a Perron-Frobenius type of property for signed matrices. Our conditions are necessary and sufficient for undirected graphs, but only sufficient for digraphs, the gap between necessity and sufficiency being filled by matrices who have this Perron-Frobenius property on the right but not on the left side (i.e., on the transpose). An exception is given by weight balanced signed digraphs, where eventual positivity corresponds to positive semidefinitness of the symmetric part of the Laplacian. Analogous conditions are obtained for signed stochastic matrices.
\end{abstract}

\section{INTRODUCTION}

Distributed algorithms for computation and control on networks often rely on a Laplacian-like dynamics to achieve their goal. The underlying assumption that is normally made is that the adjacency matrix of the graph is nonnegative. There are however situations in which one would like to include in the model of the network also negative edges. They could correspond to adversarial attacks on a communication network (e.g. an attacker flipping the sign of the transmitted value to purposively disrupt consensus) or to faulty processes in some distributed communication scheme. In DC microgrids, they are induced by negative self-loops introduced by the conductance of net loads [33] and they are of relevance in the analysis of small-disturbance angle stability [32]. More generally, negative edges are an important feature in all contexts in which collaboration among the agents of a network coexists with antagonism: social networks [15], biological networks [21], ecological networks [17], etc.

When a graph is signed, then there is more than one way to construct a signed Laplacian matrix. In particular, two main alternatives have been extensively studied in the literature. In [31] we referred to them as "opposing" and "repelling" Laplacians. The first one has on the diagonal the sum of the absolute values of the row elements [3]. Consequently, it is always diagonally dominant but may or may not have 0 as eigenvalue (the latter case happening when the graph is structurally balanced). Hence the negated Laplacian is always at least marginally stable (and Hurwitz when the graph is not structurally balanced), see [3], [4], [7], [20]. The second Laplacian, instead, has on the diagonal the sum of the row

\footnotetext{
*Work supported in part by a grant from the Swedish Research Council (grant n. 2015-04390).

C. Altafini is with the Division of Automatic Control, Department of Electrical Engineering, Linköping University, SE-58183 Linköping, Sweden. E-mail: claudio.altafini@liu.se
}

elements taken with sign, hence when there are negative weights it may fail to be diagonally dominant although it always has 0 as an eigenvalue [6], [34]. As a consequence, the marginal stability of the negated Laplacian is no longer guaranteed. This is the Laplacian studied in e.g. [6], [9], [11], [10], [36], and also considered in this paper. Conditions that guarantee its stability have been sought mostly in the undirected graph case [11], [10], [36]. For instance, [36] studied signed graphs with a single negative edge, and, by making use of the notion of effective resistance over the negative edge, obtained necessary and sufficient conditions for positive semidefinitness of the Laplacian $L$ (i.e., for marginal stability of $-L$ ). The result was generalized and reinterpreted in terms of passivity in [11] and extended to an arbitrary number of negative edges in [10]. All of these results rely heavily on the concept of effective resistance, which is univocally defined only for undirected graphs. On digraphs, in fact, various alternative notions of effective resistance are possible, emphasizing different aspects [35]. It is not clear if and how a directed effective resistance can be used to attain conditions guaranteeing stability of a nonsymmetric $-L$. Even without using effective resistance, such conditions appear very difficult to obtain, see e.g. [2], [1].

The aim of this paper is to approach the problem of investigating the stability of signed Laplacians from a different perspective, relying on recent generalizations of the Perron-Frobenius (PF) theorem [16]. If the canonical way of formulating the PF theorem (for the positive orthant) is to consider a matrix which is nonnegative or Metzler, it has been shown in [23], [25], [26] that the category of matrices enjoying a PF property (namely, spectral radius which is a simple strictly dominating real eigenvalue of the matrix, of positive eigenvector) is strictly larger that nonnegative (or Metzler) matrices, and includes also matrices having some off-diagonal entries that are negative. These matrices are called eventually positive because their powers become positive matrices after a certain exponent. Eventual positivity has been used by this author to study consensuslike problems in [6], and to represent linear systems which are externally but not internally positive in [5].

In this paper we show that indeed eventually positive matrices can provide an effective way to obtain stability conditions for (negated) signed Laplacians. The conditions we obtain are necessary and sufficient in the undirected case, but are only sufficient in the directed case. In the latter case, the gap between necessity and sufficiency admits a neat interpretation: it corresponds to matrices that obey a "right PF property" but not a "left PF property", i.e., 
such that their transpose fails to satisfy the PF property. We also show that for weight balanced digraphs our eventual positivity conditions become necessary and sufficient, and equivalent to positive semidefinitness of the symmetric part of the Laplacian.

The approach generalizes in an easy way also to discretetime systems, i.e., to matrices that are row stochastic. In fact, when a matrix has negative entries but still has row sums equal to 1, its Schur stability is no longer guaranteed [13]. In this case, the equivalent concept of eventually stochastic matrix can be defined and used in an analogous way.

For both signed Laplacians and signed stochastic matrices, the conditions we obtain are based on the computation of the dominant eigenvalue/eigenvector pair. As it is known for instance from the power method, such computation is much cheaper than a full spectral analysis. In this sense, we believe that our conditions are computationally interesting when dealing with large-scale networks.

The rest of this paper is organized as follows: in Sect. II we present the required linear-agebraic notions, and in Sect. III our conditions for stability of signed Laplacians on both undirected and directed graphs. Finally in Sect. IV the analogous conditions for stability of signed stochastic matrices are given.

\section{LINEAR ALGEBRAIC PRELIMINARIES}

Given a matrix $A=\left(a_{i j}\right) \in \mathbb{R}^{n \times n}, A \geq 0$ means elementwise nonnegative, i.e., $a_{i j} \geq 0$ for any $i, j \in$ $1, \ldots, n$, and $A \neq 0$, while $A>0$ means elementwise positive, i.e., $a_{i j}>0$ for all $i, j=1, \ldots, n$. This notation is used also for vectors. The spectrum of $A$ is denoted $\operatorname{sp}(A)=\left\{\lambda_{1}(A), \ldots, \lambda_{n}(A)\right\}$, where $\lambda_{i}(A), i=1, \ldots, n$, are the eigenvalues of $A$, and the vector space generated by its columns is $\operatorname{span}(A)$. The spectral radius of $A, \rho(A)$, is the smallest real positive number such that $\rho(A) \geq\left|\lambda_{i}(A)\right|$, $\forall i=1, \ldots, n$. A matrix $A \in \mathbb{R}^{n \times n}$ is said to be irreducible if there does not exist a permutation matrix $\Pi$ such that $\Pi^{T} A \Pi$ is block triangular, that is

$$
\Pi^{T} A \Pi \neq\left[\begin{array}{cc}
A_{11} & A_{12} \\
0 & A_{22}
\end{array}\right],
$$

where $A_{11}, A_{12}$ and $A_{22}$ are nontrivial square matrices. We say that $A$ is Hurwitz stable (resp. Schur stable) if $\operatorname{Re}\left[\lambda_{i}(A)\right]<0$ (resp. $\left|\lambda_{i}(A)\right|<1$ ) for any $i$, and it is marginally stable (resp. marginally Schur stable) if $\operatorname{Re}\left[\lambda_{i}(A)\right] \leq 0$ (resp. $\left|\lambda_{i}(A)\right| \leq 1$ ) and $\lambda_{i}(A)$ such that $\operatorname{Re}\left[\lambda_{i}(A)\right]=0$ (resp. $\left|\lambda_{i}(A)\right|=1$ ) is a simple root of the minimal polynomial of $A$. The digraph whose adjacency matrix is $A$ is indicated $\mathcal{G}(A)$. It has an edge connecting the node $j$ to the node $i$ if and only if $a_{i j} \neq 0 . \mathcal{G}(A)$ is strongly connected if and only if $A$ is irreducible. A quadratic form $x^{T} A x$ is said positive semidefinite (psd) if $x^{T} A x \geq 0 \forall x \in$ $\mathbb{R}^{n}$. The matrix $A$ is said psd if $x^{T} A x=x^{T}\left(A+A^{T}\right) x / 2$ is psd.

Given $A \geq 0$, the matrix

$$
B=s I-A \quad s>0,
$$

is called a Z-matrix. If in addition $s \geq \rho(A)$, then $B$ is called an M-matrix. In particular, an M-matrix $B$ in which $s>\rho(A)$ is nonsingular and such that $-B$ is Hurwitz stable. If instead $s=\rho(A), B$ is a singular M-matrix. If in addition $A$ is irreducible, then $-B$ is also marginally stable. Given $A$, the comparison matrix of $A$, denoted $M(A)$, has $\left|a_{i i}\right|$ on the diagonal and $-\left|a_{i j}\right|$ in the entry $(i, j), i \neq j$. A matrix $A$ is called an H-matrix if its comparison matrix $M(A)$ is an M-matrix. It is said an $H_{+}$-matrix if in addition $a_{i i} \geq 0$, $i=1, \ldots, n,[18]$.

\section{A. Perron-Frobenius property and eventual positivity}

Definition 1 A matrix $A \in \mathbb{R}^{n \times n}$ has the (strong) PerronFrobenius property if $\rho(A)$ is a simple positive eigenvalue of $A$ s.t. $\rho(A)>|\lambda|$ for every $\lambda \in \operatorname{sp}(A), \lambda \neq \rho(A)$, and $\chi$, the right eigenvector relative to $\rho(A)$, is positive.

Denote $\mathcal{P} \mathcal{F}$ the set of matrices in $\mathbb{R}^{n \times n}$ that possess the Perron-Frobenius property. $\rho(A)$ is sometimes referred to as the PF eigenvalue, and $\chi$ as the corresponding PF eigenvector. $\mathrm{PF}$ properties are naturally associated with nonnegative matrices but also with matrices having some negative entries, the so-called eventually positive matrices [16], [23], [25], [14], [6].

Definition 2 A real square matrix $A$ is said to be eventually positive if $\exists k_{o} \in \mathbb{N}$ such that $A^{k}>0$ for all $k \geq k_{0}$.

Following [27], eventually positive matrices will be denoted $A \stackrel{\vee}{>}$. Clearly, $A \stackrel{\vee}{>} 0$ implies $A$ irreducible. The following necessary and sufficient condition relates eventually positive matrices and PF property.

Theorem 1 ([25], Theorem 2.2) For $A \in \mathbb{R}^{n \times n}$ the following are equivalent:
1) Both $A, A^{T} \in \mathcal{P} \mathcal{F}_{n}$;
2) $A \stackrel{\vee}{>} 0$;
3) $A^{T} \stackrel{\vee}{>} 0$.

Notice that from Theorem 1 of [23], we have an easy test of eventual positivity: $A$ is eventually positive iff $A^{k}>0$ and $A^{k+1}>0$ for some positive integer $k$. Another computationally easy way to check the PF property is to use the power method to compute the dominant eigenvalue/eigenvector pair. Also the following lemma will be useful later on.

Lemma 1 ([6], Lemma 1) Consider $A \stackrel{\vee}{>} 0$ and denote $\chi>$ 0 its right $P F$ eigenvector. Then any eigenvector $\chi_{1}$ of $A$ such that $\chi_{1}>0$ must be a multiple of $\chi$.

\section{B. Eventually exponentially positive matrices}

Recall that a matrix $A \in \mathbb{R}^{n \times n}$ is said exponentially positive if $e^{A t}=\sum_{k=0}^{\infty} \frac{A^{k} t^{k}}{k !}>0 \forall t \geq 0$, and that $A$ is exponentially positive if and only if $A$ is essentially nonnegative, i.e., $a_{i j} \geq 0 \forall i \neq j$ [26]. 
Definition 3 A matrix $A \in \mathbb{R}^{n \times n}$ is said eventually exponentially positive if $\exists t_{o} \in[0, \infty)$ such that $e^{A t}>0 \forall t \geq t_{o}$.

The relationship between eventual positivity and eventual exponential positivity is provided by the following lemma.

Lemma 2 ([26], Thm 3.3) A matrix $A \in \mathbb{R}^{n \times n}$ is eventually exponentially positive if and only if $\exists d \geq 0$ such that $A+$ $d I \stackrel{\vee}{>} 0$.

\section{Eventually stochastic matrices}

A matrix $W$ is row stochastic if $W \mathbf{1}=\mathbf{1}, 0 \leq w_{i j} \leq 1$. Analogously, $W$ is column stochastic if $\mathbf{1}^{T} W=\mathbf{1}^{T}, 0 \leq$ $w_{i j} \leq 1$, and doubly stochastic if it is both row and column stochastic.

Definition 4 A matrix $W$ is said eventually row (resp. column) stochastic if $W \stackrel{\vee}{>} 0$ and $W \mathbf{1}=\mathbf{1}$ (resp. $\left.\mathbf{1}^{T} W=\mathbf{1}^{T}\right)$. It is said eventually doubly stochastic if it is both eventually row and column stochastic ${ }^{1}$.

Lemma 3 ([6] Lemma in Sect. VI.C) If $W$ is eventually row (or column) stochastic, then $\rho(W)=1$ is a positive eigenvalue of $W$.

\section{Stability of LAPlacians Via EVENTUALLy POSITIVE MATRICES}

Given the signed digraph $\mathcal{G}(A)$ of weighted adjacency matrix $A$, and denoted $\sigma_{i}^{\text {in }}=\sum_{j=1}^{n} a_{i j}$ the weighted indegree of node $i$, let us define the Laplacian as

$$
L=\Sigma-A \quad \text { where } \quad \Sigma=\operatorname{diag}\left(\sigma_{1}^{\text {in }}, \ldots, \sigma_{n}^{\text {in }}\right) .
$$

The Laplacian (2) is often used in the consensus problem [29], in which all agents are required to converge to the same value. If we think of a distributed control problem on $\mathcal{G}(A)$ for a system of integrators $\dot{x}_{i}=u_{i}, i=1, \ldots, n$, then $L$ can be obtained by choosing $u_{i}=-\sum_{j=1, \ldots, n} a_{i j}\left(x_{i}-x_{j}\right)$ [24], [29], or, in matrix form,

$$
\dot{x}=-L x .
$$

When $\mathcal{G}(A)$ is strongly connected and $A \geq 0, L$ is a singular irreducible M-matrix and the 0 eigenvalue has multiplicity 1. The associated right eigenvector is $\mathbf{1}$, and $\lim _{t \rightarrow \infty} x(t)=$ $x^{*} \in \operatorname{span}(\mathbf{1})$ is a consensus value for (3). This means that $-L$ is marginally stable, and that the quadratic form $x^{T} L x=$ $x^{T}\left(L+L^{T}\right) x / 2$ is psd.

The definition of Laplacian in (2) can be extended to the case in which $\mathcal{G}(A)$ is a signed graph. Since $A$ can have negative entries, $\sigma_{i}^{\text {in }}$ can even become negative. In our recent paper [31], the Laplacian (2) in presence of negative weights is referred to as "repelling signed Laplacian", terminology which allows us to distinguish it from a second signed Laplacian (referred to in [31] as "opposing signed Laplacian"),

\footnotetext{
${ }^{1}$ In the literature, instead of eventually stochastic, the terms "essentially stochastic" [22] or "somewhat stochastic" [12] are sometimes used.
}

obtained replacing $\sigma_{i}^{\text {in }}$ with $\sigma_{i}^{\text {in,abs }}=\sum_{i=1}^{n}\left|a_{i j}\right|$ [3], [7], [20].

When $A$ is signed and irreducible, then for (2) we have the following easily verifiable properties.

Proposition 1 Consider a signed, strongly connected digraph $\mathcal{G}(A)$. Then for the corresponding Laplacian (2) we have

1) 0 is always an eigenvalue of right eigenvector 1;

2) L need not be diagonally dominant;

3) $-L$ need not be marginally stable;

4) The quadratic form $x^{T} L x$ need not be psd.

Proof: Property 1 is true by construction. Properties 24 will be shown through counterexamples later on (Examples 1-2).

In particular, determining conditions guaranteeing the marginal stability of (3) is a difficult task.

\section{A. Nonnegative digraph case}

As already mentioned, $A \geq 0$ irreducible implies $-L$ marginally stable, with right eigenvector $\mathbf{1}$ associated to the eigenvalue 0 . The following result is the basis of popular Lyapunov constructions used in the consensus problem, where choosing the quadratic form $V(x)=x^{T} \Xi x$ for some positive definite $\Xi$ leads to a Lyapunov derivative of the form $\dot{V}(x)=-x^{T}\left(\Xi L+L^{T} \Xi\right) x$ [28], [37]. The uniqueness part of the following theorem is however less known (we could not find any reference in the consensus literature), hence we provide a complete proof.

Theorem 2 Consider a digraph $\mathcal{G}(A)$ with $A \geq 0$ irreducible. Let 1 and $\xi>0$ be the right and left eigenvectors of $L$ relative to the eigenvalue 0 . Then $\xi$ is the unique (up to a scalar multiplication) positive vector for which the diagonal matrix $\Xi=\operatorname{diag}(\xi)$ is s.t. $\Xi L+L^{T} \Xi$ is psd. For it, $\operatorname{ker}\left(L^{T} \Xi\right)=\operatorname{ker}(L)=\operatorname{span}(\mathbf{1})$ and hence $\operatorname{corank}\left(\Xi L+L^{T} \Xi\right)=1$.

Proof: $L$ is a singular irreducible M-matrix, and its null space $\operatorname{ker}(L)=\operatorname{span}(\mathbf{1})$ has dimension 1. Furthermore, since the diagonal entries of $L$ are all positive, it is also an $H_{+}$matrix. From Lemma 3.21-3.22 of [18] there exists a unique (up to scalar multiplication) diagonal matrix $\Delta=\operatorname{diag}\left(\delta_{1}, \ldots, \delta_{n}\right)$ such that $\operatorname{ker}\left(L^{T} \Delta\right)=\operatorname{ker}(L) \subseteq$ $\operatorname{ker}\left(\Delta L+L^{T} \Delta\right)$, while from Theorem 3.23 of [18] $L$ admits a unique (up to scalar multiplication) "Lyapunov scaling factor" $\Delta$ such that $\Delta L+L^{T} \Delta$ psd. Since $L^{T} \xi=L^{T} \Xi \mathbf{1}$, it must necessarily be $\left[\delta_{1} \ldots \delta_{n}\right]^{T}=\alpha \xi, \alpha \in \mathbb{R}$. From $\operatorname{ker}(L)=\operatorname{ker}(\Xi L)=\operatorname{ker}\left(L^{T} \Xi\right)=\operatorname{span}(\mathbf{1}), \Xi L+L^{T} \Xi$ must necessarily have corank 1 .

As consequence of Theorem 2, one has that the symmetric part of $L$ is psd only in the so-called weight balanced case. A graph $\mathcal{G}$ is said weight balanced if $\sigma_{i}^{\text {in }}=\sum_{j} a_{i j}=\sum_{j} a_{j i}=$ $\sigma_{i}^{\text {out }} \forall i=1, \ldots, n$. 
Corollary 1 Consider a digraph $\mathcal{G}(A)$ with $A \geq 0$ irreducible. $L_{s}=\left(L+L^{T}\right) / 2$ is psd of corank 1 iff $\mathcal{G}(A)$ is weight balanced.

Proof: One direction is obvious from Theorem 2. For the other one, from Lemma 6.3 of [19] we have that if $L+L^{T}$ is psd, then it must be $\operatorname{ker}(L)=\operatorname{ker}\left(L^{T}\right) \subseteq \operatorname{ker}\left(L+L^{T}\right)$. But this is true only if $\mathbf{1}$ is also the left eigenvector of $L$ relative to 0 , i.e, if $\mathcal{G}(A)$ is weight balanced.

In other words, Corollary 1 implies that whenever indegree and out-degree of $\mathcal{G}(A)$ are not identical, $L_{s}$ cannot be psd.

\section{B. Signed undirected graph case}

From Proposition 1, when $\mathcal{G}(A)$ is a signed graph, $L$ may fail to be diagonally dominant (technically, $L$ need not be an H-matrix), which may or may not lead to loss of stability of $-L$, in a way which is complicated to check.

The case of $\mathcal{G}(A)$ signed and undirected has been studied extensively in the literature, mostly in terms of the so-called effective resistance matrix. Here we express instead the condition of $L$ psd in terms of eventually positive matrices.

Theorem 3 Consider an undirected graph $\mathcal{G}(A)$ with adjacency matrix $A$ which is symmetric, irreducible and signed. Then we have that $L$ is psd of $\operatorname{corank}(L)=1$ iff $-L$ is eventually exponentially positive.

Proof: Assume $L$ is psd of corank 1 and let $0=\lambda_{1}<$ $\lambda_{2} \leq \ldots \leq \lambda_{n}$ be the spectrum of $L$. Choose $d>\frac{\lambda_{n}}{2}>0$ and consider $B=d I-L$. By construction, $\operatorname{sp}(B)$ is $d-$ $\lambda_{n} \leq \ldots \leq d-\lambda_{2}<d$ and $\rho(B)=d$ is an eigenvalue of eigenvector 1 , i.e., $B \in \mathcal{P} \mathcal{F}$, which, from Theorem 1, implies $B \stackrel{\vee}{>} 0$ and hence, from Lemma $2,-L$ is eventually exponentially positive. For the other direction, from $L \mathbf{1}=$ $d \mathbf{1}-B \mathbf{1}=0$, we see that $d$ is an eigenvalue of $B$ with eigenvector 1 . Since $B \stackrel{\vee}{>} 0$ implies that also $\rho(B)$ has a strictly positive eigenvector, from Lemma 1 , we obtain that $\rho(B)=d$ and therefore that $\mathbf{1}$ is the PF eigenvector of $B$. Since $\rho(B)$ is simple, reversing the construction above for $\operatorname{sp}(B)$ gives that all the remaining eigenvalues of $L$ must be strictly positive, i.e., $L$ must be psd of corank 1 .

\section{Signed digraph case}

In the signed digraph case, the conditions we obtain are no longer necessary and sufficient for marginal stability of $-L$.

Theorem 4 Consider a strongly connected signed digraph $\mathcal{G}(A)$, and the corresponding Laplacian (2). If - $L$ is eventually exponentially positive, then the matrix $-L$ is marginally stable and the system (3) converges to

$$
x^{*}=\lim _{t \rightarrow \infty} x(t)=\frac{\xi^{T} x_{o} \mathbf{1}}{\xi^{T} \mathbf{1}},
$$

where $\xi$ is the left eigenvector of $L$ relative to 0 . Viceversa, if $-L$ is marginally stable then $\exists$ a scalar $d \geq 0$ such that $d I-L \in \mathcal{P} \mathcal{F}$.

Proof: From Lemma 2, $-L$ eventually exponentially positive means that $\exists$ a scalar $d \geq 0$ such that $B=d I-L \stackrel{\vee}{>}$ 0 . From $L \mathbf{1}=0$, it is $B \mathbf{1}=d \mathbf{1}-L \mathbf{1}=d \mathbf{1}$, i.e., $\mathbf{1}$ is a right eigenvector of $B$ of eigenvalue $d$. If $B \stackrel{\vee}{>} 0$ then it means that $\rho(B)$ is a simple eigenvalue of strictly positive left and right eigenvectors $\chi$ and $\xi$. From Lemma 1, it must necessarily be $\chi=\alpha \mathbf{1}$ for some scalar $\alpha$, and therefore $\rho(B)=d$. Since $\rho(B)$ is simple, all other eigenvalues of $L$ must have strictly positive real part. The value of $x^{*}$ follows then straightforwardly. As for the second implication, if $-L$ is marginally stable, then $0=\lambda_{1}(L)<\operatorname{Re}\left[\lambda_{i}(L)\right]$, $i=2, \ldots, n$, and $\mathbf{1}$ is the eigenvector relative to 0 . But then, provided $d>\max _{i=2, \ldots, n} \frac{\left|\lambda_{i}(L)\right|}{2}, B=d I-L$ has $\rho(B)=d$ of eigenvector $\mathbf{1}$, hence $B \in \mathcal{P} \mathcal{F}$.

Remark 1 The gap between the two conditions of Theorem 4 corresponds to matrices $L$ s.t. $B=d I-L \in \mathcal{P} \mathcal{F}$ for some $d \geq 0$, but $B^{T} \notin \mathcal{P} \mathcal{F}$ for all $d \geq 0$. The stability of such class of Laplacians cannot be determined a priori with the tools developed in this paper, as the following two examples show.

Example 1 In correspondence of

$$
A=\left[\begin{array}{cccc}
0 & -0.7 & 0 & 0.3 \\
1.4 & 0 & -0.2 & 0.4 \\
0.7 & 0 & 0 & 2.1 \\
0 & 0 & 1.3 & 0
\end{array}\right]
$$

it is $\operatorname{sp}(L)=\{0,0.7325 \pm 0.1220 i, 3.8349\}$, i.e. $-L$ is marginally stable, even though the left eigenvector associated to 0 is not positive. For $d>1.92, B=d I-L \in \mathcal{P} \mathcal{F}$ but $B^{T} \notin \mathcal{P} \mathcal{F}$. Notice also that $L$ has both positive and negative values on the diagonal.

\section{Example 2 For}

$$
A=\left[\begin{array}{cccc}
0 & 1.9 & -0.5 & 0 \\
0 & 0 & 0 & 1 \\
-0.3 & 0.2 & 0 & 0.2 \\
1.8 & 0 & 0 & 0
\end{array}\right]
$$

it is $\operatorname{sp}(L)=\{-0.0890,0,2.1945 \pm 1.2509 i\}$ i.e. $-L$ is unstable. Clearly $x^{T} L x$ is not psd. Also in this case the left eigenvector associated to 0 is not positive, and, for $d>2.6$, $B=d I-L \in \mathcal{P} \mathcal{F}$ but $B^{T} \notin \mathcal{P} \mathcal{F}$.

The following is instead an example of eventually exponentially positive matrix.

Example 3 For

$$
A=\left[\begin{array}{cccc}
0 & 1 & 0 & 0 \\
0 & 0 & 2.6 & 0 \\
0.3 & 0 & 0 & 1.1 \\
0.9 & -0.2 & 0.9 & 0
\end{array}\right]
$$


we have $\operatorname{sp}(L)=\{0,1.6956 \pm 0.9452 i, 3.2089\}$. For $d \geq$ 1.61 it is $B=d I-L \stackrel{\vee}{>} 0$.

The case of $L$ weight balanced is an exception, as there is no gap between necessity and sufficiency.

Corollary 2 Consider a strongly connected signed digraph $\mathcal{G}(A)$ such that the corresponding Laplacian (2) is weight balanced. Then the following conditions are equivalent:

(i) $-L$ is eventually exponentially positive;

(ii) $-L$ is marginally stable;

(iii) $L_{s}=\left(L+L^{T}\right) / 2$ is psd of corank 1 .

Proof: $(i) \Longrightarrow(i i)$ : This direction is implied by Theorem 4.

$($ ii $) \Longrightarrow(i)$ : If $-L$ marginally stable it follows from the proof of Theorem 4 that for $d>\max _{i=2, \ldots, n} \frac{\left|\lambda_{i}(L)\right|}{2}>0$, $B=d I-L$ has $\rho(B)=d$ which is a simple eigenvalue of right eigenvector $\mathbf{1}$. $\mathrm{L}$ weight balanced implies $L \mathbf{1}=L^{T} \mathbf{1}=$ 0 , which means that the argument can be repeated also for $L^{T}$ leading to $B, B^{T} \in \mathcal{P} \mathcal{F}$.

(ii) $\Longrightarrow$ (iii): Split $x$ into $x=\alpha \mathbf{1}+y$ with $\alpha=\mathbf{1}^{T} x_{o} / n$ and $y \in \operatorname{span}(\mathbf{1})^{\perp}$. From marginal stability and $\operatorname{ker}(L)=$ $\operatorname{ker}\left(L^{T}\right)=\operatorname{span}(\mathbf{1})$, on $\operatorname{span}(\mathbf{1})^{\perp}, V(y)=y^{T} y / 2$ is positive definite and its derivative along the trajectories of (3) such that $\dot{V}=-y^{T}\left(L+L^{T}\right) y / 2$ negative definite, i.e., $L_{s}$ psd of corank 1 .

$($ iii $) \Longrightarrow($ ii $)$ : If follows from a well-known eigenvalue inequality (see e.g. [8], Fact 5.11.24) that

$$
0=\min _{i}\left(\lambda_{i}\left(L_{s}\right)\right) \leq \operatorname{Re}\left(\lambda_{i}(L)\right)
$$

i.e., $L$ has eigenvalues with nonnegative real part. Furthermore, from Lemma 6.3 of [19], if $L_{s}$ is psd then $\operatorname{ker}(L)=\operatorname{ker}\left(L^{T}\right) \subseteq \operatorname{ker}\left(L_{s}\right)$. Since $\operatorname{ker}\left(L_{s}\right)=\operatorname{span}(\mathbf{1})$ and $\operatorname{corank}\left(L_{s}\right)=1$, and since weight balance of $L$ implies $L \mathbf{1}=L^{T} \mathbf{1}=0$, the implication follows.

\section{StABility OF EVEnTUALly STOCHASTIC MATRICES}

For a graph $\mathcal{G}(W)$, the discrete-time equivalent of (3) is given by the system

$$
x(k+1)=W x(k) .
$$

When $W \geq 0, W$ is a row stochastic matrix with $\mathbf{1}$ as right eigenvector associated to the 0 eigenvalue. $W$ is doubly stochastic when $\mathcal{G}(W)$ is undirected. The system (4) is widely used in discrete-time consensus protocols. A closely related version of (4) (with $W$ column stochastic) is also commonly used to describe transition probabilities in Markov chains [30].

We proceed now to relax the condition $W \geq 0$, i.e., we consider $\mathcal{G}(W)$ which is a signed graph. Clearly when $W$ is not nonnegative then any probabilistic interpretation associated to the state in (4) is lost. However, if $W^{k}>0$ for $k \geq k_{o}$, any sufficiently long downsampling of the system (4) can still be considered a well-posed transition matrix, provided $W$ is eventually stochastic.

\section{A. Signed undirected graph case}

The following necessary and sufficient condition is the analogous of Theorem 3 for discrete-time systems.

Theorem 5 Consider an undirected signed graph $\mathcal{G}(W)$ with $W$ symmetric, irreducible and such that $W \mathbf{1}=\mathbf{1} . W$ is marginally Schur stable iff $W$ is eventually doubly stochastic.

Proof: $W$ marginally Schur stable and symmetric implies $\lambda_{1}(W)= \pm 1$ is a simple eigenvalue such that $\left|\lambda_{1}(W)\right|>\left|\lambda_{i}(W)\right|, i=2, \ldots, n$. Combined with $W \mathbf{1}=\mathbf{1}$, it must be $\lambda_{1}(W)=\rho(W)=1$, meaning that $W=$ $W^{T} \in \mathcal{P} \mathcal{F}$ or, from Theorem 1 , that $W$ is eventually doubly stochastic. Viceversa, $W$ eventually doubly stochastic means $W \stackrel{\vee}{>} 0$, i.e., $\rho(W)$ is a simple eigenvalue of $W$ with positive eigenvector. Since it is also $W \mathbf{1}=\mathbf{1}$, from Lemma 1 , it must be that $\rho(W)=1$ is an eigenvalue of multiplicity 1 strictly dominating all other eigenvalues. Hence $W$ is marginally Schur stable.

\section{B. Signed digraph case}

As for Laplacians on signed digraphs, the sufficiency condition for marginal stability that can be obtained from eventually stochastic matrices is in general not a necessary condition.

Theorem 6 Consider a signed digraph $\mathcal{G}(W)$ with $W$ signed and irreducible. If $W$ is eventually row stochastic, then it is marginally Schur stable. Viceversa, if $W$ marginally Schur stable and such that $W \mathbf{1}=\mathbf{1}$ then $W \in \mathcal{P} \mathcal{F}$.

Proof: $W$ eventually row stochastic implies that $W \mathbf{1}=$ 1 and, since $W$ irreducible and $W \stackrel{\vee}{>} 0, \lambda_{1}(W)=\rho(W)=$ 1 must be a simple eigenvalue. Hence $\left|\lambda_{i}(W)\right|<1, i=$ $2, \ldots, n$, meaning that $W$ is marginally Schur stable. As for the other direction, if $W$ irreducible and such that $W \mathbf{1}=$ $\mathbf{1}$ is marginally Schur stable, it means that $\left|\lambda_{i}(W)\right|<1$, $i=2, \ldots, n$, i.e., $\rho(W)=1$ is a simple strictly dominant eigenvalue of eigenvector 1 . Hence $W \in \mathcal{P} \mathcal{F}$.

The following example shows that the condition of eventual stochasticity in Theorem 6 is not necessary for marginal stability.

Example 4 The matrix

$$
W=\left[\begin{array}{cccc}
0.0893 & 0.8036 & -0.2679 & 0.375 \\
0.9932 & 0.0068 & 0 & 0 \\
0.8625 & -0.0375 & 0.1 & 0.075 \\
0.114 & 0.8547 & 0 & 0.0313
\end{array}\right]
$$

is such that $W \mathbf{1}=\mathbf{1}$. Since $\operatorname{sp}(W)=\{-0.4603 \pm$ $0.3218 i, 0.148,1\}, W$ is marginally Schur stable. Furthermore, $W \in \mathcal{P} \mathcal{F}$, but $W^{T} \notin \mathcal{P} \mathcal{F}$, hence $W$ is not an eventually stochastic matrix.

The following example shows instead that the weaker condition $W \in \mathcal{P} \mathcal{F}$ does not suffice for marginal stability. 
Example 5 The matrix

$$
W=\left[\begin{array}{cccc}
0.0368 & 0 & 0.659 & 0.3042 \\
0 & 0.0872 & 3.1947 & -2.2819 \\
0 & 0.9895 & 0.0105 & 0 \\
0.9322 & 0 & 0 & 0.0678
\end{array}\right]
$$

is such that $W \mathbf{1}=\mathbf{1}, \operatorname{sp}(W)$

$=$ $\{-1.5494,-0.8928,1,1.6447\}, \quad$ meaning that $\rho(W)=1.6447$ of right eigenvector $\nu=$ $\left[\begin{array}{llll}0.2303 & 0.8242 & 0.4991 & 0.1362\end{array}\right]^{T}>0 . \quad$ Therefore $W \in \mathcal{P} \mathcal{F}$, but $W$ unstable. Notice that $W$ has two positive right eigenvectors and obviously $W^{T} \notin \mathcal{P} \mathcal{F}$.

As for signed Laplacians, also for signed stochastic matrices the weight balanced case (here $W \mathbf{1}=W^{T} \mathbf{1}=\mathbf{1}$ ) leads instead to a necessary and sufficient condition.

Corollary 3 Consider a strongly connected signed digraph $\mathcal{G}(W)$ such that $W$ is weight balanced. Then $W$ eventually doubly stochastic iff it is marginally Schur stable.

The proof is analogous to that of Corollary 2 .

\section{CONCLUSiOnS}

The conditions provided in this paper for checking the stability of signed Laplacians are more general than those available in the literature (they hold also for digraphs) and are computationally interesting, as they require only the calculation of the dominant eigenpair of $\mathrm{L}$, which is more efficient than computing the entire spectrum of $L$.

What remain to understand is the relationship between our eventual positivity property and the notion of effective resistance used in the literature for similar purposes. We believe that expressing eventual positivity in terms of effective resistance (or viceversa) could lead to significant insight into the structure of signed Laplacians.

\section{REFERENCES}

[1] S. Ahmadizadeh, I. Shames, S. Martin, and D. Nesic. Corrigendum to "on eigenvalues of Laplacian matrix for a class of directed signed graphs" [linear algebra appl. 523 (2017) 281-306]. Linear Algebra and its Applications, 530:541 - 557, 2017.

[2] S. Ahmadizadeh, I. Shames, S. Martin, and D. Nesic. On eigenvalues of Laplacian matrix for a class of directed signed graphs. Linear Algebra and its Applications, 523:281 - 306, 2017.

[3] C. Altafini. Consensus problems on networks with antagonistic interactions. IEEE Trans. on Automatic Control, 58(4):935-946, 2013.

[4] C. Altafini. Stability analysis of diagonally equipotent matrices. Automatica, 49(9):2780-2785, 2013.

[5] C. Altafini. Minimal eventually positive realizations of externally positive systems. Automatica, 68:140-147, 2016.

[6] C. Altafini and G. Lini. Predictable dynamics of opinion forming for networks with antagonistic interactions. Automatic Control, IEEE Transactions on, 60(2):342-357, Feb 2015.

[7] F. Belardo. Balancedness and the least eigenvalue of Laplacian of signed graphs. Lin. Alg. Appl., 446:133 - 147, 2014.

[8] D. Bernstein. Matrix Mathematics: Theory, Facts, and Formulas with Application to Linear Systems Theory. Princeton Univ. Press, 2005.

[9] J. Bronski and L. DeVille. Spectral theory for dynamics on graphs containing attractive and repulsive interactions. SIAM Journal on Applied Mathematics, 74(1):83-105, 2014.
[10] W. Chen, J. Liu, Y. Chen, S. Z. Khong, D. Wang, T. Basar, L. Qiu, and K. H. Johansson. Characterizing the positive semidefiniteness of signed Laplacians via effective resistances. In 2016 IEEE 55th Conf Decision and Control (CDC), pp. 985-990, Dec 2016.

[11] Y. Chen, S. Z. Khong, and T. T. Georgiou. On the definiteness of graph Laplacians with negative weights: Geometrical and passivitybased approaches. In 2016 American Control Conference (ACC), pages 2488-2493, July 2016.

[12] B. Ćurgus and R. I. Jewett. Somewhat stochastic matrices. ArXiv e-prints, Sept. 2007.

[13] S. Eger. On limits of powers of certain absolutely row-stochastic matrices. Linear Algebra and its Applications, 508:1 - 13, 2016.

[14] A. Elhashash and D. B. Szyld. On general matrices having the PerronFrobenius property. ELA. The Electronic Journal of Linear Algebra [electronic only], 17:389-413, 2008.

[15] G. Facchetti, G. Iacono, and C. Altafini. Exploring the low-energy landscape of large-scale signed social networks. Phys. Rev. E 86:036116, 2012.

[16] S. Friedland. On an inverse problem for nonnegative and eventually nonnegative matrices. Israel J. of Mathematics, 29(1):43-60, 1978.

[17] G. Giordano and C. Altafini. Qualitative and quantitative responses to press perturbations in ecological networks. Sci. Rep., 7:11378, 2017.

[18] D. Hershkowitz and H. Schneider. Lyapunov diagonal semistability of real H-matrices. Lin. Alg. Appl., 71:119 - 149, 1985.

[19] D. Hershkowitz and H. Schneider. Scalings of vector spaces and the uniqueness of Lyapunov scaling factors. Linear and Multilinear Algebra, 17(3-4):203-226, 1985.

[20] Y. Hou, J. Li, and Y. Pan. On the Laplacian eigenvalues of signed graphs. Linear and Multilinear Algebra, 51(1):21-30, 2003.

[21] G. Iacono and C. Altafini. Monotonicity, frustration, and ordered response: an analysis of the energy landscape of perturbed large-scale biological networks. BMC Syst. Biol., 4:83, 2010.

[22] E. Johnsen. Essentially doubly stochastic matrices i. elements of the theory over arbitrary fields. Linear Algebra and its Applications, 4(3):255 - 282, 1971.

[23] C. R. Johnson and P. Tarazaga. On matrices with Perron-Frobenius properties and some negative entries. Positivity, 8(4):327-338, 2004.

[24] M. Mesbahi and M. Egerstedt. Graph-theoretic Methods in Multiagent Networks. Princeton University Press, 2010.

[25] D. Noutsos. On Perron-Frobenius property of matrices having some negative entries. Lin. Alg. Appl., 412:132 - 153, 2006.

[26] D. Noutsos and M. Tsatsomeros. Reachability and holdability of nonnegative states. SIAM Journal on Matrix Analysis and Applications, 30(2):700-712, 2008.

[27] D. D. Olesky, M. J. Tsatsomeros, and P. van den Driessche. $M_{\vee}$ matrices: a generalization of M-matrices based on eventually nonnegative matrices. ELA. The Electronic Journal of Linear Algebra, 18:339-351, 2009.

[28] Z. Qu. Cooperative Control of Dynamical Systems: Applications to Autonomous Vehicles. Springer London, 2009.

[29] W. Ren, R. Beard, and E. Atkins. Information consensus in multivehicle cooperative control. Control Systems, IEEE, 27(2):71 -82, 2007.

[30] E. Seneta. Non-negative Matrices and Markov Chains. Springer Series in Statistics. Springer, 2006.

[31] G. Shi, C. Altafini, and J. Baras. Dynamics over signed networks. SIAM Review, 61(2), 229-257, 2019.

[32] Y. Song, D. J. Hill, and T. Liu. Small-disturbance angle stability analysis of microgrids: A graph theory viewpoint. In 2015 IEEE Conference on Control Applications (CCA), pages 201-206, Sep. 2015.

[33] Y. Song, D. J. Hill, and T. Liu. Local stability of de microgrids: A perspective of graph laplacians with self-loops. In 2017 IEEE 56th Annual Conference on Decision and Control (CDC), pages 2629-2634, Dec 2017.

[34] L. Xiao and S. P. Boyd. Fast linear iterations for distributed averaging. Systems \& Control Letters, pages 65-78, 2004.

[35] G. F. Young, L. Scardovi, and N. E. Leonard. A new notion of effective resistance for directed graphs-part i: Definitions and properties. IEEE Transactions on Automatic Control, 61(7):1727-1736, July 2016.

[36] D. Zelazo and M. Bürger. On the definiteness of the weighted Laplacian and its connection to effective resistance. In 53rd IEEE Conference on Decision and Control, pages 2895-2900, Dec 2014.

[37] H. Zhang, F. L. Lewis, and Z. Qu. Lyapunov, adaptive, and optimal design techniques for cooperative systems on directed communication graphs. IEEE Transactions on Industrial Electronics, 59(7):30263041, July 2012. 